\title{
Decoupling Nonclassical Nonlinear Behavior of Elastic Wave Types
}

\author{
Marcel C. Remillieux, ${ }^{1, *}$ Robert A. Guyer, ${ }^{1,2}$ Cédric Payan, ${ }^{3}$ and T. J. Ulrich ${ }^{1}$ \\ ${ }^{1}$ Geophysics Group (EES-17), Los Alamos National Laboratory, Los Alamos, New Mexico 87545, USA \\ ${ }^{2}$ Department of Physics, University of Nevada, Reno, Nevada 89577, USA \\ ${ }^{3}$ Laboratoire de Mécanique et d'Acoustique, LMA CNRS UPR 7051, Aix-Marseille Université, 13402 Marseille Cedex 20, France
}

(Received 23 December 2015; published 17 March 2016)

\begin{abstract}
In this Letter, the tensorial nature of the nonequilibrium dynamics in nonlinear mesoscopic elastic materials is evidenced via multimode resonance experiments. In these experiments the dynamic response, including the spatial variations of velocities and strains, is carefully monitored while the sample is vibrated in a purely longitudinal or a purely torsional mode. By analogy with the fact that such experiments can decouple the elements of the linear elastic tensor, we demonstrate that the parameters quantifying the nonequilibrium dynamics of the material differ substantially for a compressional wave and for a shear wave. This result could lead to further understanding of the nonlinear mechanical phenomena that arise in natural systems as well as to the design and engineering of nonlinear acoustic metamaterials.
\end{abstract}

DOI: 10.1103/PhysRevLett.116.115501

Nonlinear mesoscopic elastic materials [1] exhibit unique and interesting properties related to nonlinear and nonequilibrium dynamics that are relevant to various natural and industrial processes ranging in scales and applications, e.g., the onset of earthquakes and avalanches in geophysics [2-4], the aging of infrastructures in civil engineering [5,6], the failure of mechanical parts in industrial settings [7-9], bone fragility in the medical field [10-12], or the design of novel materials, including nonlinear metamaterials, for shock absorption, acoustic focusing, and energy-harversting systems [13]. These properties include the dependence of wave speed and damping parameters on strain amplitude [5,14,15], slow relaxation [16,17], and hysteresis with end-point memory [18-20]. Consolidated (see the work referenced previously) and unconsolidated granular media [21-24] are of particular interest for laboratory-scale experiments because they can provide reference measurements to study or engineer these properties. The latter, when consisting of disorded bead pack or granular crystal lattices, serves as a simplified paradigm for understanding the key mechanisms responsible for nonequilibrium dynamics whereas the former provides a more complex but faithful representation of realistic systems.

In consolidated granular media, nonequilibrium dynamics is thought to originate from the microscopic-sized imperfections (e.g., microcracks, debonding at interfaces, grain contacts, etc.) in the "soft" bond system that connects together mesoscopic-sized "hard" elements (e.g., grains or crystals) [25], with experimental evidence recently

Published by the American Physical Society under the terms of the Creative Commons Attribution 3.0 License. Further distribution of this work must maintain attribution to the author(s) and the published article's title, journal citation, and DOI. presented for thermally damaged samples of concrete [26]. These micro- and mesoscopic features are typically distributed throughout the sample and affect its dynamic response at a macroscopic scale through a process of homogenization, thus offering a rich multiscale problem in material physics. Nonequilibrium dynamics has been quantified experimentally in nonlinear mesoscopic elastic materials, through the nonclassical nonlinear elastic parameter $\alpha$, by resonant experiments in which a slender bar with free boundary conditions, representing a one-dimensional unconstrained system, is vibrated with a harmonic signal at one end while the elastic response is recorded at the opposite end $[27,28]$. The source signal sweeps a frequency range around a resonance frequency at a fixed drive level and the experiment is repeated with signals of increasing amplitudes so that the variation of the resonance frequency can be tracked as a function of the maximum strain in the sample. Typically, such experiments are conducted around the frequency of the fundamental mode of longitudinal vibration, thus limiting the analysis to a narrow frequency range and one particular term of the elastic tensor. In other words, it is not clear how nonequilibrium dynamics is affected by the mode order (e.g., $n$th longitudinal mode) and the mode type (e.g., longitudinal, torsional, and bending modes). Payan et al. [29] recently tried to address this question and demonstrated experimentally that the parameter $\alpha$ was indeed dependent on the mode type. However, the study did not link this parameter with the terms of the elastic tensor because the resonant modes used in the analysis included coupled contributions from compressional and shear waves, as a result of the sample geometry deviating too much from a 1D system. In a 1D system made of an isotropic material, the longitudinal mode of vibration is related to the Young modulus $E$ and so may be used to quantify the parameters of nonequilibrium 
dynamics, $\alpha_{E}$. Likewise, if one is able to vibrate the sample in a pure torsional mode, the parameter $\alpha_{G}$ corresponding to the shear modulus $G$ can be determined. The objective of this Letter is to take a first step into extending the theory of nonequilibrium dynamics in granular materials to the full elastic tensor (as opposed to simply one term) and over a wide frequency range.

A series of two resonance experiments was conducted on a cylindrical sample of Berea sandstone (Cleveland Quarries, Amherst, Ohio) with a diameter of $25.8 \mathrm{~mm}$, a length of $305.5 \mathrm{~mm}$, a mass density of $2054 \mathrm{~kg} / \mathrm{m}^{3}$, and a nominal permeability ranging between 500 and $1000 \mathrm{mD}$. The sample instrumented with transducers is shown in Fig. 1. The linear elastic properties of this sample were characterized in previous work using resonant ultrasound spectroscopy [30]. It was found that, at the relatively low frequencies considered in this study, the sample is well described by a homogeneous and isotropic material with $E=9.9 \mathrm{GPa}$ and $G=4.6 \mathrm{GPa}$. Such an assumption is valid because the variability of the mass density and elastic properties at a mesoscopic scale is homogenized at a macroscopic scale. In the first experiment, longitudinal motion was induced in the sample with a compressional piezoelectric disc (a PZT-5H ceramic with a diameter of $25.54 \mathrm{~mm}$ and a thickness of $6.35 \mathrm{~mm}$ ) glued with epoxy onto one flat end of the sample. Meanwhile, the longitudinal $\left(v_{x}\right)$ component of the particle velocity was recorded with an out-of-plane laser vibrometer (Polytec OFV-303) on the opposite free end, where the spatial maximum is expected to be reached for all modes. In the second experiment, five shear piezoelectric plates (PZT-4 ceramic with dimensions of $15 \times 15 \times 1 \mathrm{~mm}^{3}$ ) were glued on the round surface of the sample, near the end opposite to the compressional transducer. In this particular configuration and when driven in phase, these shear plates can induce efficiently a torsional motion in the sample. Meanwhile, the vertical $\left(v_{y}\right)$ component of the particle velocity was recorded with an in-plane laser vibrometer (Polytec OFV-552) on the round surface, close to the opposite end. Note that, at the particular sensing position indicated in Fig. 1, $v_{y}$ in Cartesian coordinates is equivalent to $v_{\theta}$ in cylindrical coordinates, which is the coordinate system chosen to treat the torsional motion. In both experiments, the transducers were driven with harmonic voltage signals generated by a function generator (National Instrument PXI-5406) and amplified 50 times by a voltage amplifier (TEGAM 2350). The signals were played for $40 \mathrm{~ms}$ at a given frequency, in frequency steps of $5 \mathrm{~Hz}$, until the desired frequency range of the experiment was spanned. For each frequency, transient vibrational responses were recorded during the last $30 \mathrm{~ms}$ of the source signal, to ensure that steady state conditions had been reached. Fast Fourier transforms of these harmonic responses were then computed to construct vibrational spectra for 19 different drive levels where the voltage imposed to the transducers was varied from 10 to $400 \mathrm{~V}$. In the first experiment, pure longitudinal modes could be excited up to the seventh order, thereby covering a frequency range of 2 to $24 \mathrm{kHz}$. The configuration of transducers used in the second experiment broke the symmetry of this system, and pure torsional modes could not be excited beyond the third order.
EXPERIMENT 1: Longitudinal Modes

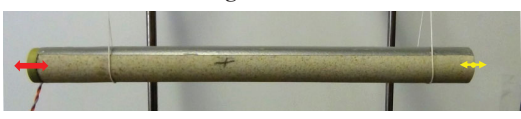

EXPERIMENT 2: Torsional Modes
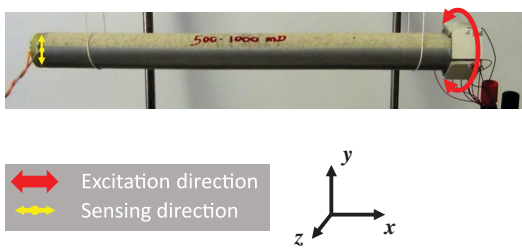

ALL MODES
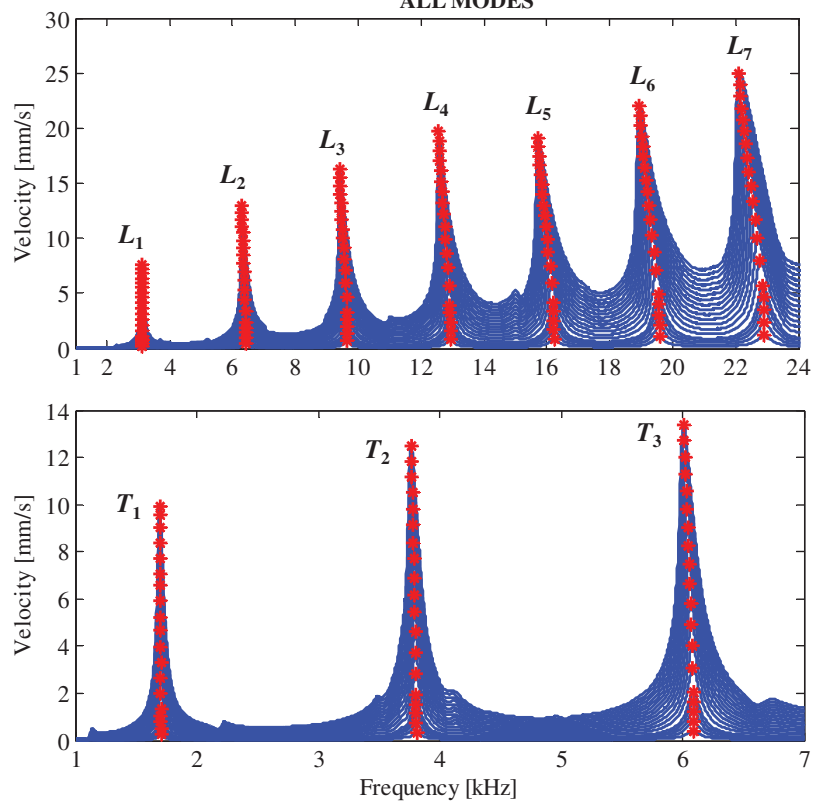

ONE MODE
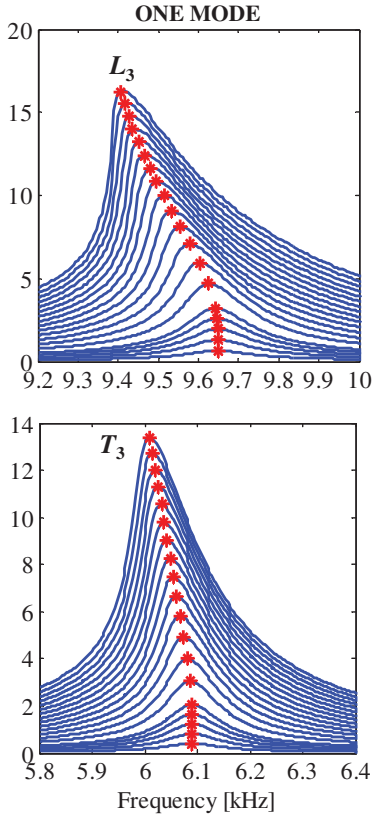

FIG. 1. Measuring vibrational spectra on a resonant bar of Berea sandstone excited with a nearly pure longitudinal motion (top) and a pure torsional motion (bottom). Experimental setup with sample and transducers (left), vibrational spectra up to the highest order mode that could be excited in a pure manner (center), and vibrational spectra for one particular mode (right). 
As shown (in Fig. 1), the expected material softening is observed in both experiments when the drive level becomes sufficiently large. For a given drive level, such softening can be quantified by plotting the relative frequency shift as a function of maximum strain in the sample, the slope of which is the parameter $\alpha$. As described in the Supplemental Material [31], additional experiments were conducted with a 3D laser vibrometer (Polytec PSV-3D-500) to measure the velocity and strain fields on the surface of the sample and establish relationships between these quantities for all modes. For the longitudinal mode, the strain component of interest is $\epsilon_{x x}$. It was found experimentally that the ratio $v_{x}^{\max } / \epsilon_{x x}^{\max }$ ranged between 1890 and $2060 \mathrm{~m} / \mathrm{s}$ for the first seven longitudinal modes. Theoretically, this ratio corresponds to the speed of sound in the sample, expressed as $c_{L}=2 L f_{1}$, where $L$ is the length of the sample and $f_{1}$ is the frequency of the fundamental longitudinal mode. Based on $L=305.5 \mathrm{~mm}$ and $f_{1}=3136 \mathrm{~Hz}$ (measured at the lowest drive amplitude), it is found that $c_{L}=1916 \mathrm{~m} / \mathrm{s}$, which falls within the values found experimentally. A similar approach was followed for the torsional modes, leading to experimental values of the $v_{\theta}^{\max } / \epsilon_{x \theta}^{\max }$ ratios ranging between 1202 and $1270 \mathrm{~m} / \mathrm{s}$. The experimental values of the $v^{\max } / \epsilon^{\max }$ ratios were used to infer the value of the maximum strain in the sample at resonance and quantify the material softening. As depicted in Fig. 2(a), the relative frequency shift varies almost linearly with the maximum strain beyond $4 \mu \epsilon$ for all longitudinal modes but the fundamental mode, for which the drive amplitude is not sufficiently large. More importantly, it appears that material softening converges to a single value (all curves superimpose) of $\alpha_{E}=3880 \pm 150$ for the modes $L_{2}$ through $L_{7}$. The same convergence property is observed for torsional modes [see Fig. 2(b)]. This first result demonstrates that in
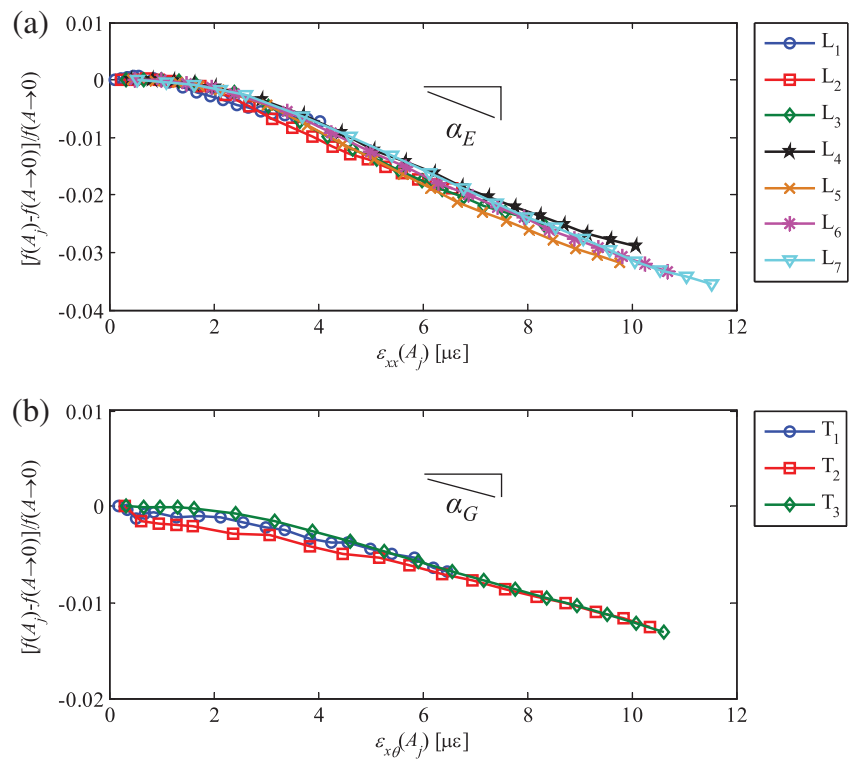

FIG. 2. Relative frequency shift as a function of strain. (a) Longitudinal modes. (b) Torsional modes. a resonant bar experiment, any mode order can be selected to quantify material softening as long as the mode type is unchanged. There is also a practical advantage in using higher order modes due to the fact that the relatively small transducers used in the experiments can inject more energy into the system at higher frequencies (see the vibrational spectra in Fig. 1). Material softening in torsional modes is much smaller, with $\alpha_{G}=1530 \pm 125$ for the modes $T_{2}$ and $T_{3}$. This value should be corrected to account for the fact that the strain component $\epsilon_{x \theta}$ is not uniform across the diameter of the sample (unlike the strain component $\epsilon_{x x}$ in a longitudinal mode). Theoretically, $\epsilon_{x \theta}$ is maximum on the surface $(r=R)$ and zero along the axis of symmetry of the sample $(r=0)$. Suppose that $\epsilon_{x \theta}=1$ at $r=R$; then the average strain over the cross-sectional area is $2 / 3$. If material softening in the torsional mode is corrected for this strain distribution, we find that $\alpha_{G}^{\text {corrected }}=2290 \pm 150$, which still deviates substantially from the value associated with the longitudinal modes.

The above experimental observations reduce to two independent 1D systems: a system of compressional modes and a system of torsional modes, to which we can associate an equation of motion of the bar and a corresponding Preisach-Mayergoyz (PM) space. The PM-space formalism [32] was first adapted to the problem of nonequilibrium dynamics in geomaterials by Guyer and McCall [33] but has been limited thus far to scalar (as opposed to tensorial) applications. The equation of motion and boundary conditions for the longitudinal modes of the bar of length $L$ $(-L / 2 \leq x \leq L / 2)$ and mass density $\rho$ can be expressed as

$$
\rho \frac{\partial^{2} u_{x}}{\partial t^{2}}=\frac{\partial}{\partial x}\left[E(x) \frac{\partial u_{x}}{\partial x}\right], \quad \epsilon_{x x}( \pm L / 2)=0,
$$

where $u_{x}$ is the displacement of the bar in the $x$ direction (the displacement is independent of $y$ and $z$ ) and $E(x)$ is the Young modulus, which varies with $x$ due to the presence of hysteretic elastic elements that depend on the strain $\epsilon_{x x}=\partial u_{x} / \partial x$. There is a PM space associated with elastic elements that respond to compressional forces in the $x$ direction, the $L$-PM space. The modes of the system will be found using the lumped element procedure sketched by Guyer and Johnson [34]. In order to use this procedure it is necessary to know $E(x)$. Supposing that the PM space has a uniform density of hysteretic elastic elements at low strain, this elastic modulus, at each point in the sample, can be expressed in terms of the maximum strain at that point as

$$
E(x)=E_{0}\left[1-\alpha_{E} \epsilon_{x x}^{\max }(x)\right],
$$

where $\epsilon_{x x}^{\max }(x)$ is the maximum strain at $x$ and $\alpha_{E}$ is the constant characterizing the elastic element density in the $L$-PM space. Here, for conciseness, we consider the fundamental resonance mode with the displacement node at the origin. Integrating Eq. (1) from 0 to $L / 2$ yields 


$$
\rho \frac{\partial^{2} U_{x}(t)}{\partial t^{2}}=-\frac{2}{L} E_{0}\left[1-\alpha_{E} \epsilon_{x x}^{\max }(0)\right] \epsilon_{x x}(0, t),
$$

where $U_{x}$ is the longitudinal displacement averaged over the half length ( 0 to $L / 2)$ of the bar. This is an equation for the motion of the right-hand side of the bar in terms of the forces at the bar center. To find $U_{x}$ and $\epsilon_{x x}(0)$, we use the analytical expression for the modal deformation of the fundamental longitudinal mode with maximum displacement amplitude $A$ driven at angular frequency $\omega$

$$
u_{x}(x, t)=A \sin \frac{\pi x}{L} e^{-i \omega t} .
$$

It follows that

$$
\begin{aligned}
U_{x}(t) & =\frac{2}{\pi} A e^{-i \omega t}, \\
\epsilon_{x x}(0, t) & =\frac{\pi}{L} A e^{-i \omega t}, \\
\bar{\epsilon}_{x x} & =\frac{2}{L} \int_{0}^{L / 2} \epsilon_{x x} d x=\frac{2}{L} u_{x}^{\max }(+L / 2) .
\end{aligned}
$$

Substitution of these three equations into Eq. (3) gives

$$
\omega^{2}=c_{L}^{2}\left[1-\hat{\alpha}_{E} \bar{\epsilon}_{x x}\right] k^{2},
$$

where $\hat{\alpha}_{E}=\alpha_{E}(\pi / 2), k=\pi / L$, and $c_{L}^{2}=E_{0} / \rho$. We have introduced $\bar{\epsilon}_{x x}$ into this equation in order to express the answer in terms of the average strain in the bar (which is not uniformly strained) and in terms of measurable quantities.

Likewise, there is a PM space associated with elastic elements that respond to torsional forces in the $\theta$ direction, the $T$-PM space. The starting point is the equation of motion and boundary conditions for the torsional modes of the bar

$$
\rho \frac{\partial^{2} u_{\theta}}{\partial t^{2}}=\frac{\partial}{\partial x}\left[G(r, x) \frac{\partial u_{\theta}}{\partial x}\right], \quad \epsilon_{x \theta}( \pm L / 2)=0,
$$

where the displacement $u_{\theta}$ in the $\theta$ direction now depends on $x$ and $r$ and $G(r, x)$ is the shear modulus, which varies also with $x$ and $r$ due to the presence of hysteretic elastic elements that depend on the strain $\epsilon_{x \theta}$. The methodology is essentially the same as above, with the difference that the displacement now depends on both $x$ and $r$. The shear modulus can be written as

$$
G(r, x)=G_{0}\left[1-\alpha_{G} \epsilon_{x \theta}^{\max }(r, x)\right],
$$

where $\epsilon_{x \theta}^{\max }(r, x)$ is the maximum strain at $(r, x)$ and $\alpha_{G}$ is the constant characterizing the elastic element density in the $T$-PM space. After carrying out the $x$-integration step, we obtain a set of equations similar to Eqs. (3)-(7). However, because of the difference in the strain as a function of $r$ in the present case, there are details involving numerical factors of order 1. Eventually, the following equation is derived for torsional modes:

$$
\omega^{2}=c_{T}^{2}\left[1-\hat{\alpha}_{G} \bar{\epsilon}_{x \theta}\right] k^{2},
$$

where $\hat{\alpha}_{G}=\alpha_{G}(\pi / 2)(9 / 8)$ and $c_{T}^{2}=G_{0} / \rho$.

The two dispersion relations (8) and (11) are of the same form. They involve the two sound velocities $c_{L}$ and $c_{T}$, the associated average strain fields $\bar{\epsilon}_{x x}$ and $\bar{\epsilon}_{x \theta}$, and the associated PM space densities $\alpha_{E}$ and $\alpha_{G}$. In addition the average strain fields are related to measured quantities quite simply.

In this Letter, we demonstrated, quantified, and described mathematically the tensorial nature of the nonequilibrium dynamics in nonlinear mesoscopic elastic materials. Since the parameters quantifying nonequilibrium dynamics increase in magnitude with the concentration of microscopic imperfections in the soft bond system, this important result leads to further questions. It would be interesting, for instance, to determine if the parameters $\alpha_{E}$ and $\alpha_{G}$ vary at different rates with environmental conditions. We can also wonder about the sensitivity of these parameters to the orientation of microfractures in a material. If so, the ability to measure the tensorial components of the nonequilibrium dynamics could lead to an increased understanding of the relationship between the evolution of the microscopic features, in particular the imperfections, and the dynamic response of the material at a macroscopic scale. Last, the tensorial features evidenced in this Letter could be used to enhance the design of nonlinear metamaterials with a wide range of applications [13].

This work was funded by the U.S. Department of Energy, Fuel Cycle R\&D, Used Fuel Disposition (Storage) campaign. We are grateful to J. A. Ten Cate, P. A. Johnson, and P.-Y. Le Bas at Los Alamos National Laboratory for fruitful discussions.

*mcr1@lanl.gov

[1] R. A. Guyer and P. A. Johnson, Phys. Today 52, No. 04, 30 (1999).

[2] P. A. Johnson and X. Jia, Nature (London) 437, 871 (2005).

[3] A. A. Delorey, K. Chao, K. Obara, and P. A. Johnson, Sci. Adv. 1, 1 (2015).

[4] V. Y. Zaitsev, P. Richard, R. Delannay, V. Tournat, and V. E. Gusev, Europhys. Lett. 83, 64003 (2008).

[5] M. Bentahar, H. El Aqra, R. El Guerjouma, M. Griffa, and M. Scalerandi, Phys. Rev. B 73, 014116 (2006).

[6] C. Payan, V. Garnier, J. Moysan, and P. A. Johnson, J. Acoust. Soc. Am. 121, EL125 (2007).

[7] I. Y. Solodov and B. A. Korshak, Phys. Rev. Lett. 88, 014303 (2001). 
[8] Y. Ohara, T. Mihara, R. Sasaki, T. Ogata, S. Yamamoto, Y. Kishimoto, and K. Yamanaka, Appl. Phys. Lett. 90, 011902 (2007).

[9] J. N. Potter, A. J. Croxford, and P. D. Wilcox, Phys. Rev. Lett. 113, 144301 (2014).

[10] M. Muller, A. Sutin, R. Guyer, M. Talmant, P. Laugier, and P. A. Johnson, J. Acoust. Soc. Am. 118, 3946 (2005).

[11] G. Renaud, S. Callé, J.-P. Remenieras, and M. Defontaine, Int. J. Nonlinear Mech. 43, 194 (2008).

[12] S. Haupert, S. Guérard, F. Peyrin, D. Mitton, and P. Laugier, PLoS One 9 (2014).

[13] M. A. Porter, P. G. Kevrekidis, and C. Daraio, Phys. Today 68, No. 11, 44 (2015).

[14] K. Winkler, A. Nur, and M. Gladwin, Nature (London) 277, 528 (1979).

[15] J. A. TenCate, E. Smith, and R. A. Guyer, Phys. Rev. Lett. 85, 1020 (2000).

[16] J. A. TenCate, D. Pasqualini, S. Habib, K. Heitmann, D. Higdon, and P. A. Johnson, Phys. Rev. Lett. 93, 065501 (2004).

[17] J. A. TenCate, Pure Appl. Geophys. 168, 2211 (2011).

[18] N. G. W. Cook and K. Hodgson, J. Geophys. Res. 70, 2883 (1965).

[19] D. J. Holcomb, J. Geophys. Res. 86, 6235 (1981).

[20] K. E. Claytor, J. R. Koby, and J. A. TenCate, Geophys. Res. Lett. 36, L06304 (2009).

[21] T. Brunet, X. Jia, and P. A. Johnson, Geophys. Res. Lett. 35, L19308 (2008).
[22] C. Inserra, V. Tournat, and V. Gusev, Appl. Phys. Lett. 92, 191916 (2008).

[23] C. J. Olson Reichhardt, L. M. Lopatina, X. Jia, and P. A. Johnson, Phys. Rev. E 92, 022203 (2015).

[24] J. Cabaret, P. Béquin, G. Theocharis, V. Andreev, V. E. Gusev, and V. Tournat, Phys. Rev. Lett. 115, 054301 (2015).

[25] L. A. Ostrovsky and P. A. Johnson, Riv. Nuovo Cimento Soc. Ital. Fis. 24, 1 (2001).

[26] C. Payan, T. J. Ulrich, P. Y. Le Bas, M. Griffa, P. Schuetz, M. C. Remillieux, and T. A. Saleh, Appl. Phys. Lett. 104, 144102 (2014).

[27] P. A. Johnson, B. Zinszner, and P. N. Rasolofosaon, J. Geophys. Res. 101, 11553 (1996).

[28] J. A. Ten Cate and T. J. Shankland, Geophys. Res. Lett. 23, 3019 (1996).

[29] C. Payan, T. J. Ulrich, P. Y. Le Bas, T. Saleh, and M. Guimaraes, J. Acoust. Soc. Am. 136, 537 (2014).

[30] M. C. Remillieux, T. J. Ulrich, C. Payan, J. Rivière, C. R. Lake, and P.-Y. Le Bas, J. Geophys. Res.: Solid Earth 120, 4898 (2015).

[31] See Supplemental Material at http://link.aps.org/ supplemental/10.1103/PhysRevLett.116.115501 for measurement of the longitudinal and torsional mode shapes using a 3D laser vibrometer.

[32] I. D. Mayergoyz, J. Appl. Phys. 57, 3803 (1985).

[33] R. A. Guyer, K. R. McCall, and G. N. Boitnott, Phys. Rev. Lett. 74, 3491 (1995).

[34] R. A. Guyer and P. A. Johnson, Nonlinear Mesoscopic Elasticity (Wiley-VCH, New York, 2009). 